\title{
Modified Kocher-Langenbeck Approach for the Treatment of Posterior Wall or Column Acetabular Fractures: The One-Incision Two-Window Method
}

\section{Tien-Yu Yang}

Chiayi Chang Gung Memorial Hospital https://orcid.org/0000-0003-4685-0310

\section{Po-Yao Chuang}

Chiayi Chang Gung Memorial Hospital

Tsan-Wen Huang

Chiayi Chang Gung Memorial Hospital

Kuo-Chin Huang ( $\nabla$ kc2672@gmail.com )

Chang Gung University College of Medicine https://orcid.org/0000-0002-3225-1504

Technical advance

Keywords: one-incision two-window method, posterior wall acetabular fracture, posterio column acetabular fracture, modified Kocher-Langenbeck approach

Posted Date: September 10th, 2020

DOI: https://doi.org/10.21203/rs.3.rs-58691/v1

License: (c) (i) This work is licensed under a Creative Commons Attribution 4.0 International License. Read Full License 


\section{Abstract}

Background: The Kocher-Langenbeck (K-L) approach is the standard method for the treatment of posterior wall or column acetabular fractures. This approach allows direct access to the posterior structures of the acetabulum, but is limited cranially and caudally by the neurovascular bundle. The present study was conducted to assess the quality of reduction and the incidence of complications in patients who underwent the modified "one-incision two-window" K-L approach.

Methods : This is a Retrospective case series with recruited thirteen consecutive patients from 2015 to 2017 who sustained an acute, displaced posterior wall or column acetabular fracture. All patients were treated with modified "one-incision two-window" K-L approach.

Results: The mean operation time, mean intraoperative blood loss, and mean incision wound length were estimated to be $103.8 \mathrm{~min}(60-120 \mathrm{~min}), 373.1 \mathrm{~mL}(100-700 \mathrm{~mL})$, and $9.7 \mathrm{~cm}(8.0-13.0 \mathrm{~cm})$, respectively. The radiographic quality of reduction was graded as anatomical reduction (maximum residual displacement $[M R D] \leq 2 \mathrm{~mm}$ ) in all cases, according to Matta's criteria. Concerning the incidence of complications, there were no iatrogenic neurovascular injury, no surgical site infections, and no osteonecrosis of the femoral head or heterotopic ossification in this cohort. One patient with transverse posterior wall fracture (group 1) experienced deep vein thrombosis in the lesion leg. Another two patients with solitary posterior wall fracture (group 2) developed posttraumatic osteoarthritis, with one diagnosed as Tonnis grade I lesion and the other as Tonnis grade III lesion. With respect to the clinical treatment outcome, the mean visual analogue scale (VAS), mean modified Harris Hip Score (mHHS) and subjective satisfaction rate were 1.7 (1.0-2.0), 90.6 (81-100), and 84.6\% (80\%-90\%), respectively. Although there was no significant difference in the satisfaction rate $(82.9 \%$ vs. $87.0 \%, P=0.941)$ at 12 months after surgery, group 1 patients had more increased VAS score (2.0 vs. $1.2, \mathrm{P}=0.016)$ and more decreased $\mathrm{mHHS}(87.7$ vs. $94.6, P=0.014)$ than group 2 patients.

Conclusions: Our pilot study confirmed that the "one-incision two-window" K-L approach is a simple, safe, reliable, and effective way to manage acute, displaced posterior wall or column acetabular fractures.

Level of evidence: Therapeutic study, level IV.

\section{Full Text}

This preprint is available for download as a PDF.

\section{Figures}


A

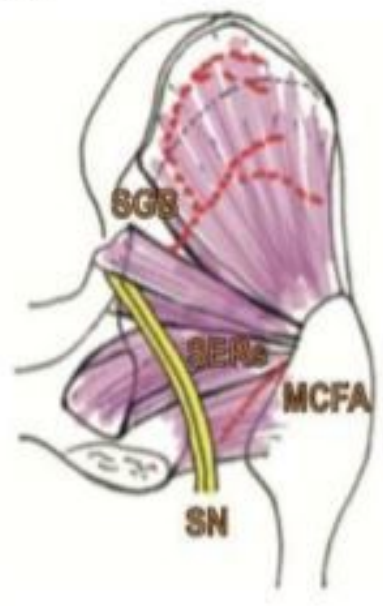

B

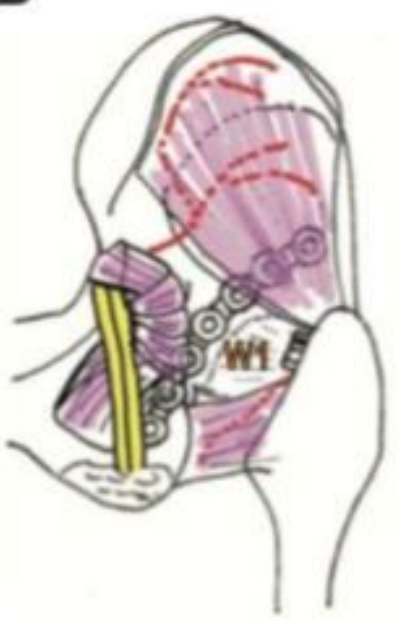

C

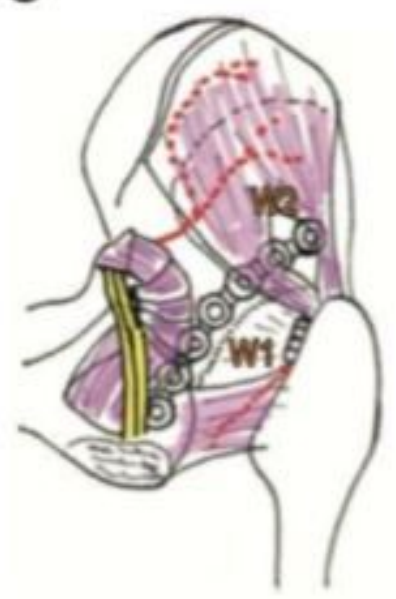

\section{Figure 1}

The modified "one-incision two-window" Kocher-Langenbeck surgical approach. (A) Posterior structures of the right hip joint. (B) Window 1 (W1) is used for controlling the posterior structures by gently elevating the short external rotators (SERs) as a flap, which preserves the medial circumflex femoral artery (MCFA) and protects the sciatic nerve (SN). After provisional reduction and fixation, a precontoured long pelvic reconstruction plate can be slid under the gluteal muscles, in line with the posterior rim of the acetabulum, spanning from the ischium to the superior wall area. Through this window, the distal end of the plate is fixed with two screws into its concave bend. (C) Window 2 (W2) is used for governing the superior wall area by splitting the gluteal muscles, which avoids vigorous retraction and prevents superior gluteal neurovascular bundle (SGB) injury. Through this window, the proximal plate screws can be applied accurately and safely in direct view. 